\title{
Dormance des bourgeons apicaux du frêne (Fraxinus excelsior L.) : évaluation du pool des nucléosides triphosphates et éventail des températures actives sur le débourrement des bourgeons en période de dormance
}

\author{
P. BARNOLA, Suzanne LAVARENNE, M. GENDRAUD \\ avec la collaboration technique de Nicole JALLUT \\ Université de Clermont II, Laboratoire de Phytonorphogenèse U.A. 45 \\ C.N.R.S., 4, rue Ledru, F 63038 Clermont-Ferrand Cedex
}

\section{Résumé}

La dormance des bourgeons des végétaux ligneux est étudiée habituellement à l'aide de la technique des boutures de nxuds isolés. Elles sont placées dans des enceintes climatisées dont les tcmpératures s'échelonnent de 5 "C à $30^{\circ} \mathrm{C}$. Plus un bourgeon est dormant plus l'éventail des températures où il manifeste de la croissance est fermé. Il n'est ouvert qu'aux températures élevées. La sortie de dormance est caractérisée par son ouverture vers les températures fraîches et basses. Cette notion conçue par Vegis (1964) s'est révélée fructueuse dans l'étude de la dormance des arbres et des buissons. Mais la technique biologique employće est critiquable. L'une des critiques, la plus importante, est que la durée des essais est suffisamment longue pour induire dans la bouture un état physiologique nouveau, différent de l'état physiologique au moment du prélèvement.

Nous proposons une technique biochimique nouvelle baséc sur les modifications de la régulation de l'énergétique cellulaire, en rapport avec l'état de dormance. L'analyse consiste à mesurer l'aptitude du matériel végétal à augmenter sa teneur en nucléosides tri-phosphates non adćnyliques (NTP) à la suite de l'apport d'un précurseur exogène de l'ATP, l'adénosine. Les résultats de cette étude sont que les conceptions de VEGIs, pour la première fois, sont développées biochimiquement. Les optimums de température de croissance des bourgeons apicaux de frêne à la fin de la période de dormance sont mieux précisés ce qui n'a jamais été faut jusqu’à présent.

\section{Introduction}

Il est classique d'évaluer la dormance des végétaux ligneux en observant l'aptitude à croître d'une population de bourgeons isolés avec un fragment de l'axe qui les porte. La vigne (Huglin, 1958 ; Pouget, 1963 ; Nigond, 1967), le pommier (Crabbé, 1968 ; Sochet, 1973 ; Zanette, 1981), le merisier (Arias \& CrabÉ, 1975), le peuplier (SOUIDI, 1976; Jourdan, 1980; REgNARd, 1984), le frêne, le tilleul (Lavarenne, Champagnat, Barnola, 1976), le framboisier, la ronce, le sureau, le noisetier (Barnola, 1976), la bourdaine (Meng-Horn, 1975), le noyer (Mauget, 1976), le pêcher (Monet-Bastard, 1971 ; Rageau, 1978), le châtaignier (Solignat, 1977 ; 
Si-Mohamed, 1983), l'abricotier (Legave, 1980), le chêne (Crochet, 1984) ont été étudiés par cette technique. Il est admis qu'un bourgeon est d'autant plus dormant que la gamme des températures auxquelles il croît est plus restreinte. Aux mois d'octobre, de novembre et de décembre, seules les températures de $22^{\prime \prime}$ à $30^{\circ} \mathrm{C}$ permettent une croissance dont la première manifestation décelable est le gonflement du bourgeon suivi de son débourrement. A la fin de l'hiver, aux mois de février et de mars, le déboturrement est possible à des températures plus basses, $8{ }^{\circ} \mathrm{C}, 12^{\circ} \mathrm{C}$ ou $15^{\circ} \mathrm{C}$ selon les espèces. En d'autres termes, l'éventail des températures compatibles avec la croissance s'est ouvert au cours de la levée de dormance. La notion d'éventail de températures en relation avec la croissance, due à Vegrs (1964), appliquée aux végétaux ligneux, s'est révélée remarquablement fructueuse. Elle a permis de montrer que l'intensité de la dormance des bourgeons de végétaux ligneux des climats tempérés dépend de leur posilion sur l'axe (Crabbé, 1968 ; BArnola, 1970). Le bourgeon apical est le plus dormant.

La technique des boutures de nœuds isolés n'est pas exempte de critiques (Barnola, 1976; Champagnat, 1983; Regnard, 1984). Le critère de croissance pris en compte, le débourrement, est imprécis et la durée des essais, qui peut atteindre 1 mois, est suffisamment longue pour induire dans la bouture un état physiologique nouveau, différent de l'état physiologique au moment du prélèvement. La dormance du bourgeon est évaluée sur une bouture comportant un fragment d'axe et non sur un bourgcon isolé de toute corrélation. Il apparaît done souhaitable de recourir à une autre méthode d'étude de la dormance des bourgeons.

L'analyse des modifications de la régulation de l'énergétique cellulaire, en rapport avec l'état de dormance, est utilisée chez le topinambour (GENDraud, 1977), le frêne (Lavarenne et al., 1982), l'embryon de pommier (Thomas et al., 1985). Cette analyse consiste à mesurer l'aptitude du matériel végétal à augmenter sa tencur en nucléosides tri-phosphates non adényliques (NTP) à la suite de l'apport d'un précurseur exogène de l'ATP, l'adénosine. Les nucléosides tri-phosphates non adényliques sont indispensables aux biosynthèses liées à la croissance, telles que les synthèses d'acides nucléiques, de protéines, de composés membranaires et pariétaux. Une élévation de la teneur en NTP consécutive à un traitement par l'adénosine révèle une capacité acerue du matériel à orienter son énergie vers les processus de croissance caractéristiques de la non dormance.

La présente note a pour objet de montrer, d'une part, qu'il est possible par un test biochimique de préciser la notion d'éventail des températures de Vegis (1964) et, d'autre part d'apporter des renseignements nouveaux sur l'évolution des bourgeons pendant la dormance.

\section{Matériel et méthodes}

\subsection{Matériel végétal}

Les bourgeons apicaux de frêne (Fraxinus excelsior L.) sont prélevés dans la nature aux mois de septembre, de décembre et de mars, de telle sorte que puissent être étudiés les stades de pré-dormance, de dormance et de post-dormance (VEgis, 1964 ; Pouget, 1963). 


\subsection{Test biologique}

Lors de chaque prélèvement, des boutures de nœuds isolés constituées du bourgeon et du fragment d'axe qui le porte, coupé à une longueur de $5 \mathrm{~cm}$, sont placées par groupe de 20 en terrines contenant un mélange tourbe-sable $(1 / 2-1 / 2)$ maintenu constamment humide. Les terrines sont mises en chambre climatisée obscure à $8{ }^{\circ} \mathrm{C}, 16^{\circ} \mathrm{C}$ et $25^{\circ} \mathrm{C}$. Les bourgeons sont observés quotidiennement et le débourrement éventuel de chacun d'entre eux est noté.

\subsection{Test biochimique}

Il suit immédiatement la récolte des bourgeons. Dix-huit bourgeons séparés de tout fragment d'axe sont placés pendant $12 \mathrm{~h}$ à $8^{\circ} \mathrm{C}, 16^{\circ} \mathrm{C}$ ou $25^{\circ} \mathrm{C}$ à l'obscurité au contact d'une solution d'adénosine $10 \mathrm{mM}$. Les échantillons témoins sont incubés dans les mêmes conditions sur de l'eau distillée. A la fin de l'incubation, les écailles sont éliminées et le matériel végétal est traité selon la méthode de Keppler et al. (1970) adaptée aux conditions expérimentales. Toutes les opérations sont effectuées à $2{ }^{\circ} \mathrm{C}$; les tissus sont broyés dans $\mathrm{HClO}_{4} 0,3 \mathrm{H}(100 \mu \mathrm{l}$ pour $10 \mathrm{mg}$ de matière fraîche) et l'ensemble est centrifugé à $12000 \mathrm{~g}$ pendant 2 minutes. Le surnageant acido-soluble est recueilli et neutralisé par $\mathrm{KHCO}_{3}$ cristallisé. Après une nouvelle centrifugation, le surnageant acido-soluble neutralisé constitue l'extrait biologique dans lequel sont dosés l'ATP et les NTP.

Le dosage enzymatique de l'ATP en présence du système luciférineluciférase est réalisé d'après PraDET. Le milieu réactionnel d'un volume final de $200 \mu$ renferme une quantité d'extrait biologique apportant entre 0 et 10 picomoles d'ATP diluée dans un tampon tris- $\mathrm{H}_{2} \mathrm{SO}_{4} 40 \mathrm{mM}, \mathrm{pH} 7,5, \mathrm{MgSO}_{4} 3,5 \mathrm{mM}, \mathrm{K}_{2} \mathrm{SO}_{4} 25 \mathrm{mM}$, EDTA $0,55 \mathrm{mM}$ additionné d'ADP $(250$ picomoles $/ 200 \mu \mathrm{l})$. La réaction de bioluminescence est déclenchée par l'injection de $50 \mu 1$ d'un mélange luciférine-luciférase (Boehringer ATP-CLS) et l'intensité lumineuse émise est mesurée à l'aide d'un photomultiplicateur «pico-ATP ». Un témoin interne renfermant une quantité d'ATP supplémentaire et connue est toujours réalisé.

Les NTP sont dosés enzymatiquement grâce à l'addition au milieu réactionnel précédent de nucléoside di-phosphate kinase (Boehringer $25 \mu g$ pour $200 \mu \mathrm{l}$ de milieu réactionnel). L'émission de lumière après addition du mélange luciférine-luciférase permet le dosage de la teneur totale en nucléosides tri-phosphates (ATP + NTP) de l'extrait. La teneur en NTP est alors calculée par différence.

Les résultats sont exprimés en picomoles de nucléosides tri-phosphates (ATP ou NTP) par mg de matière fraîche. Il s'agit dans chaque cas de valeurs moyennes pour lesquelles sont précisées les étendues de dispersion. Les expériences ont été faites deux années successives. Les teneurs en nucléosides ne sont pas exactement les mêmes pour deux cycles de dormance consécutifs mais les différences entre $8{ }^{\circ} \mathrm{C}$, $16{ }^{\circ} \mathrm{C}$ et $25^{\circ} \mathrm{C}$ subsistent. Aussi seule est représentée l'évolution allant du mois de septembre 1983 au mois de mars 1984. 


\section{Résultats}

3.1. Bourgeons prélevés au mois de septembre

Le test des boutures de nouds isolés (fig. 1a) révèle que seuls quelques bourgeons placés à 25 "C ( 3 sur 20 ) débourrent après un temps de latence important. A 16 "C et $8^{\circ} \mathrm{C}$, aucune évolution morphologique n'est décelable.

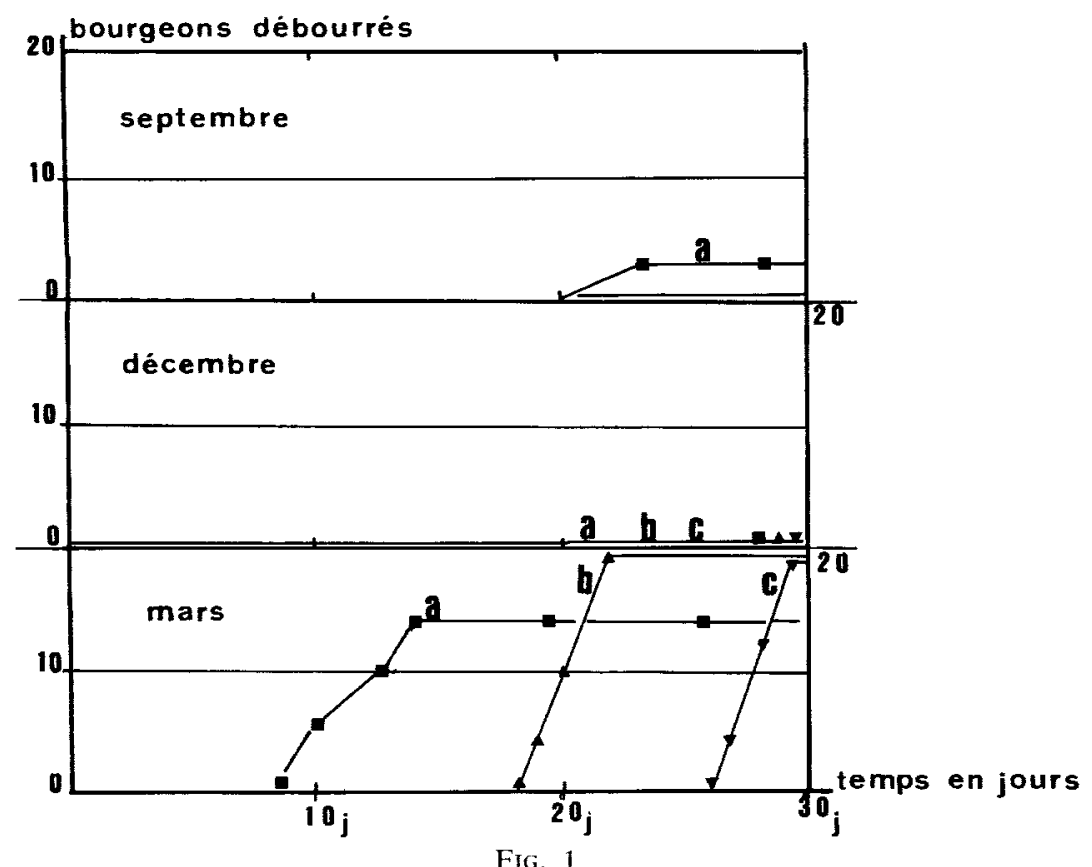

Débourrements de 20 bourgeons apicaux de frênes de 3 ans prélevés dans les conditions naturelles en boutures de nauds isolés placées respectivement à $25^{\circ} \mathrm{C}\left( \pm 1^{\circ} \mathrm{C}\right): a, 16^{\circ} \mathrm{C}\left( \pm 1{ }^{\circ} \mathrm{C}\right): b, 8{ }^{\circ} \mathrm{C}\left( \pm 1^{\circ} \mathrm{C}\right): c$.

Opening of 20 apical buds of 3 year old ash, sampled as isolated node cuttings in natural conditions and placed at $25{ }^{\circ} \mathrm{C}\left( \pm 1{ }^{\circ} \mathrm{C}\right): a, 16^{\circ} \mathrm{C}\left( \pm 1{ }^{\circ} \mathrm{C}\right): \mathrm{b}$, and $8{ }^{\circ} \mathrm{C}\left( \pm 1{ }^{\circ} \mathrm{C}\right):$ e, respectively.

L'évolution du pool des nucléosides tri-phosphates des bourgeons consécutive à l'incubation sur adénosine dépend essentiellement de la température à laquelle est effectuée l'incubation (fig. 2) :

- dans les bourgeons placés à $25^{\circ} \mathrm{C}$ au contact du nucléoside, l'augmentation du pool nucléotidique concerne uniquement l'ATP (figure 2a). Il apparaît même une diminution de la teneur en NTP. Si l'incubation est réalisée avec des bourgeons partiellement carencés par un séjour préalable de 5 jours sur papier filtre imbibé d'eau, le résultat est tout à fait différent puisque le pool de NTP s'accroît alors notablement 
(figure 2b). Ce résultat est à rapprocher de celui de Gendraud (1977), obtenu avec le topinambour. L'interprétation donnée suggère qu'une température d'incubation élevée ne permet pas l'accumulation des NTP par suite de leur utilisation très rapide dans les processus de croissance. La carence préalable aurait pour effet de réduire suffisamment ces processus pour révéler l'accumulation de NTP ;

- dans les bourgeons placés à $16^{\circ} \mathrm{C}$ sur la solution d'adénosine, les résultats obtenus confirment ceux de LAVARENne et al. (1982). Les pools d'ATP et ne NTP augmentent et cette augmentation est immédiatement visible car la température d'incubation limite la croissance (figure $2 \mathrm{c}$ ) ;

- à $8{ }^{\circ} \mathrm{C}$, aucune modification des teneurs en nucléosides tri-phosphates n'est provoquée par le contact avec le nucléoside (figure $2 \mathrm{~d}$ ).

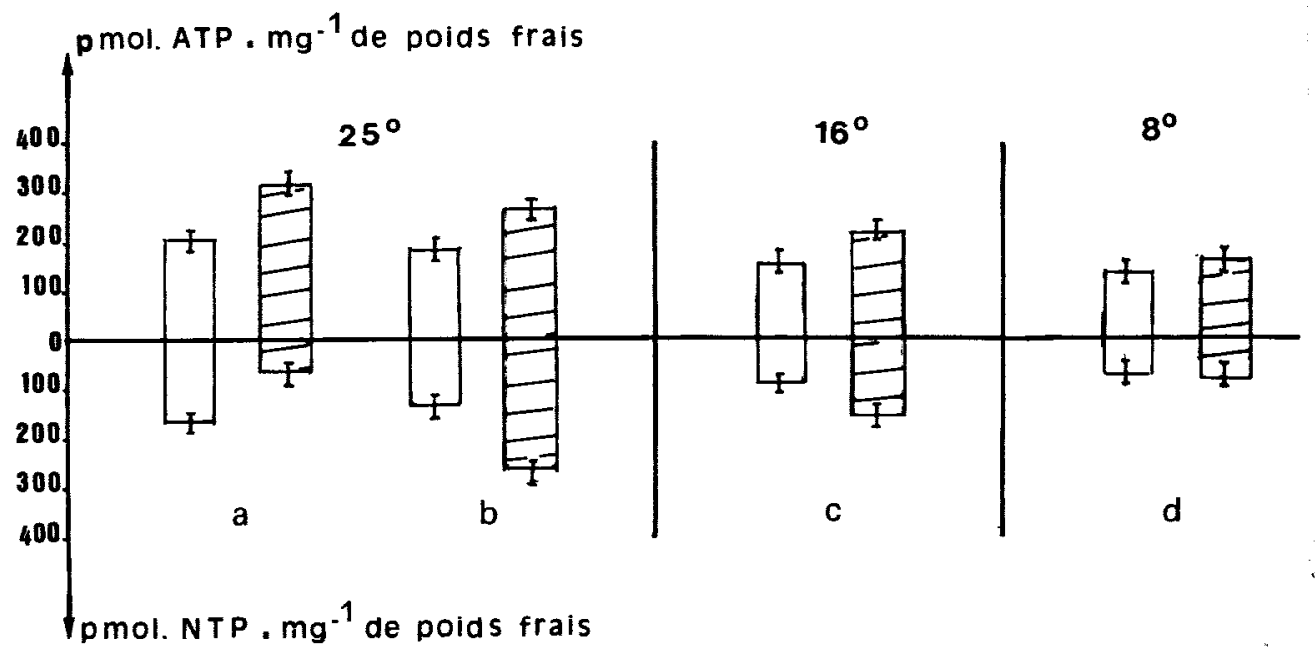

FIG. 2

Evaluation des nuclésides tri-phosphates de 18 bourgeons apicaux de frênes de 3 ans prélevés dans les conditions naturelles (jardin du laboratoire) au mois de septembre.

The evaluation of nucleotide triphosphates in 18, 3 year old apical ash buds, sampled in natural conditions (laboratory garden), in september.

Incubation des bourgeons / Buds incubated:

$\square$ sur eau / on water.

sur adénosine / on adenosine.

a : à $25^{\circ} \mathrm{C}\left( \pm 1^{\circ} \mathrm{C}\right) / \mathrm{a}:$ at $25^{\circ} \mathrm{C}\left( \pm 1^{\circ} \mathrm{C}\right)$.

b : à $25^{\circ} \mathrm{C}\left( \pm 1{ }^{\circ} \mathrm{C}\right)$ après carence partielle en sucre $/ \mathrm{b}:$ at $25^{\circ} \mathrm{C}\left( \pm 1^{\circ} \mathrm{C}\right)$ with partial sugar deficiency.

c : à $16^{\circ} \mathrm{C}\left( \pm 1{ }^{\circ} \mathrm{C}\right) / c$ : at $16^{\circ} \mathrm{C}\left( \pm l^{\circ} \mathrm{C}\right)$.

$\mathrm{d}$ : à $8^{\circ} \mathrm{C}\left( \pm 1^{\circ} \mathrm{C}\right) / d$ : at $8^{\circ} \mathrm{C}\left( \pm 1^{\circ} \mathrm{C}\right)$.

$\mathrm{I}$ : étendue de dispersion des mesures / I : range of values.

Selon les critères adoptés pour l'interprétation du test biochimique (Gendraud, 1977 ; Lavarenne et al., 1982; Thomas et al., 1985) il apparait qu'au mois de septembre, les bourgeons apicaux présentent des aptitudes énergétiques à la croissance à $25^{\circ} \mathrm{C}$ et $16^{\circ} \mathrm{C}$ bien que celle-ci ne se réalise pas, ou de manière sporadique, sur les boutures de nouds isolés. 


\subsection{Bourgeons prélevés au mois de décembre}

A $25^{\circ} \mathrm{C}, 16^{\circ} \mathrm{C}$ et $8^{\circ} \mathrm{C}$ aucun bourgeon appartenant aux boutures de nœuds isolés effectuées au mois de décembre ne manifeste le moindre signe de débourrement pendant les 30 jours que dure le test (figure 1). Les teneurs des bourgeons en NTP, après incubation sur la solution d'adénosine à $25^{\circ} \mathrm{C}, 16^{\circ} \mathrm{C}$ ou $8{ }^{\circ} \mathrm{C}$ ne sont pas modifiées par rapport aux témoins et une carence partielle préalable à l'incubation ne modifie pas notablement ces résultats (fig. 3). Enfin, les pools nucléotidiques sonı moins importants que ceux des échantillons du mois de septembre. Pour les bourgeons récoltés au mois de décembre les données fournies par les deux tests biochimiquc et biologique concordent et indiquent une incapacité totale à croître, révélant la dormance profonde du bourgeon apical.

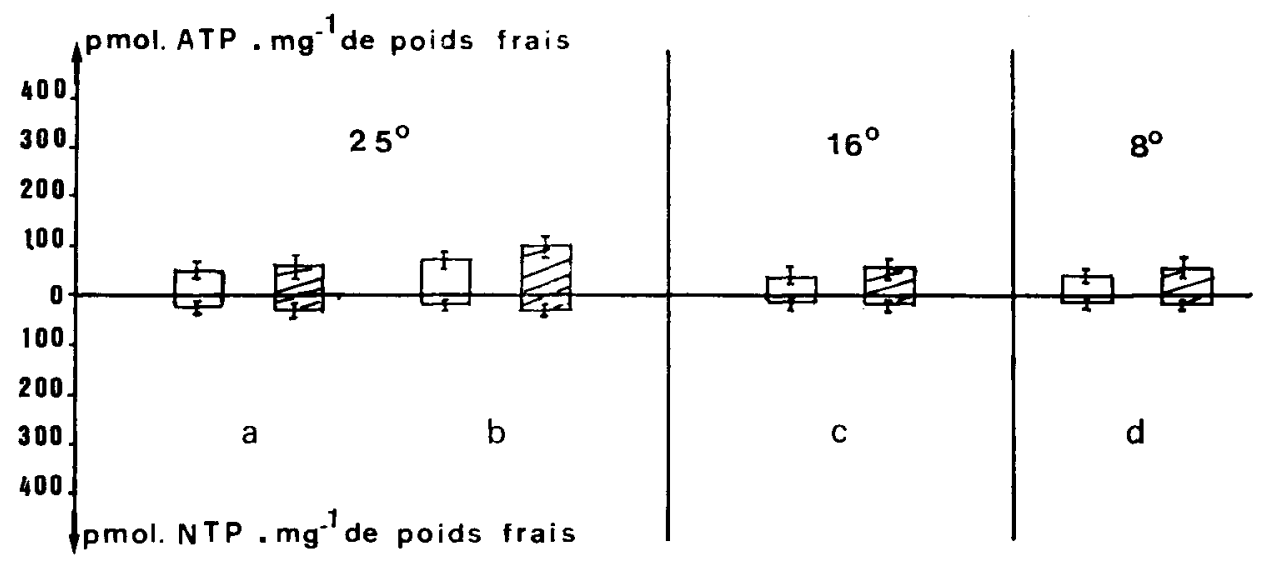

FIG. 3

Evaluation des nucléosides tri-phosphates en décembre (mêmes légondes qué pour la fig. 2).

The evaluation of nucleotides triphosphates in December (same key as for fig. 2).

\subsection{Bourgeons prélevés au mois de mars}

Dans les conditions naturelles la période de prélèvement précède de 4 à 5 semaines le débourrement. Placés en boutures de nœuds isolés, la majorité des bourgeons débourre aux trois températures choisies (fig. 1). Le temps de latence est plus lent à $8^{\circ} \mathrm{C}$ qu'à $16^{\circ}$ et $25^{\circ} \mathrm{C}$, et à cette dernière température, l'irrégularité de la pente de la courbe de débourrement ainsi que le nombre de bourgeons ayant débourré, inférieurs au maximum possible, laissent supposer une perturbation dans la physiologie des échantillons.

A 25 "C, l'incubation des bourgeons sur l'adénosine ne produit que peu ou pas de modification dans le pool nucléotique et la situation n'est pas améliorée par une carence partielle préalable (fig. $4 \mathrm{~b}$ ). A $16^{\circ} \mathrm{C}$ et 8 " $\mathrm{C}$ au contraire cette carencc permet de mettre en évidence une forte capacité du matériel à accroître l'ensemble des teneurs en nucléosides tri-phosphates (fig. 4d), la différence enregistrée entre les résultats obtenus à $25^{\circ} \mathrm{C}$ d'une part et aux températures plus basses, d'autre part, mérite d'être soulignée. 


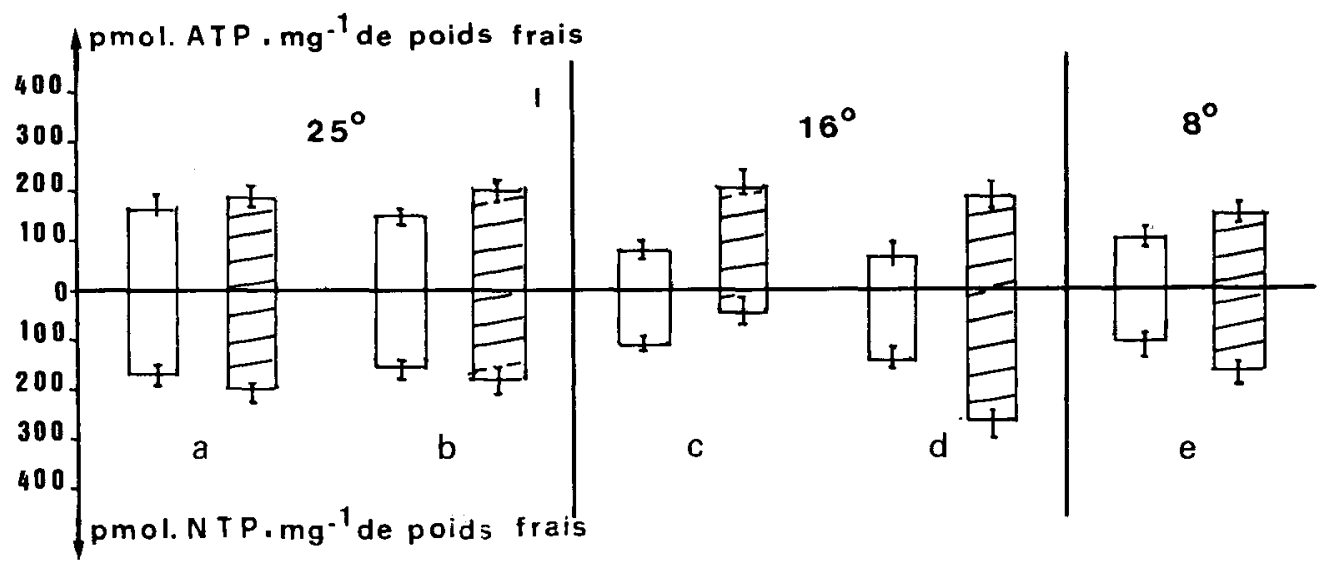

FIG. 4

Evaluation des nucléosides tri-phosphates en mars.

The evaluation of nucleotide triphosphates in March.

Incubation des bourgeons / Buds incubated:

$\square$ sur eau / on water.

日ur adénosine / on adenosine.

a : à $25^{\circ} \mathrm{C}\left( \pm 1{ }^{\circ} \mathrm{C}\right) /$ a : at $25^{\circ} \mathrm{C}\left( \pm 1{ }^{\circ} \mathrm{C}\right)$.

$\mathrm{b}$ : à $25^{\circ} \mathrm{C}\left( \pm 1^{\circ} \mathrm{C}\right)$ après carence particlle en sucre $/ \mathrm{b}$ : at $25^{\circ} \mathrm{C}\left( \pm 1^{\circ} \mathrm{C}\right)$ with partial sugar deficiency.

c: à $16^{\circ} \mathrm{C}\left( \pm 1{ }^{\circ} \mathrm{C}\right) / \mathrm{c}$ : at $16^{\circ} \mathrm{C}\left( \pm 1{ }^{\circ} \mathrm{C}\right)$.

$\mathrm{d}$ : à $16^{\circ} \mathrm{C}\left( \pm 1{ }^{\circ} \mathrm{C}\right)$ après carence partielle en sucre $/ d$ : at $16^{\circ} \mathrm{C}\left( \pm 1^{\circ} \mathrm{C}\right)$ with partial sugar deficiency.

e : à $8{ }^{\circ} \mathrm{C}\left( \pm 1{ }^{\circ} \mathrm{C}\right) /$ e: at $8^{\circ} \mathrm{C}\left( \pm l^{\circ} \mathrm{C}\right)$.

$\mathrm{I}$ : étendue de dispersion des mesures / I : range of values.

Ainsi, au mois de mars, la dormance des bourgeons apicaux est levée bien que, dans la nature, le débourrement n'apparaisse qu'au mois d'avril; il est possible que ce retard soit lié à l'approvisionnement en eau des territoires aptes à croître (CotTignies, 1983).

\section{Discussion et conclusions}

\subsection{Eventail des températures et dormance}

Sur bouture de nouds isolés la capacité de croissance des bourgeons apicaux de frêne dépend de la date de leur récolte dans la nature. Très réduite au mois de septembre, nulle au mois de décembre elle est restaurée au mois de mars. L'éventail des températures permettant la croissance apparaît déjà relativement fermé à l'automne, période d'entrée en dormance. Il est largement ouvert au printemps, 4 à 5 semaines avant le débourrement sur l'arbre. A cette époque la dormance est 
éliminée et le frêne se trouve en situation de post-dormance (VEgIs, 1964; Pouget, 1963). Une telle évolution est commune aux végétaux ligneux des régions tempérées comme le montre l'analyse des résultats rappelés en introduction.

Les conséquences d'une incubation des bourgeons sur une solution d'adénosine dépendent elles aussi de la date du prélèvement dans la nature. Au mois de septembre, elle entraîne l'accumulation de NTP à $25^{\circ} \mathrm{C}$ et $16^{\circ} \mathrm{C}$ mais non à 8 " $\mathrm{C}$. Au mois de décembre, le traitement est inefficace alors qu'au mois de mars son action nette à $16^{\circ} \mathrm{C}$ et 8 " $\mathrm{C}$ est réduite à $25^{\circ} \mathrm{C}$. En d'autres termes la réactivité des bourgeons à l'automne, à l'entrée en dormance, est située dans l'éventail allant de $16^{\circ} \mathrm{C}$ à $25^{\circ} \mathrm{C}$. Elle est nulle en hiver, au maximum de la dormance, et au printemps, en période de post-dormance, elle est importante à $8^{\circ} \mathrm{C}$ et $16^{\circ} \mathrm{C}$. En phase de pré-dormance, au mois de septembre, l'éventail des températures auxquelles le métabolisme nucléotidique peut être modifié par l'adénosine est ouvert sur des valeurs élevées, alors qu'en post-dormance, au mois de mars, cette ouverture correspond à des températures moyennes ou fraîches. Il est possible que les différences enregistrées dans les modalités de débourrement des bourgeons en pré et postdormance aient leur origine dans les caractéristiques membranaires du matériel végétal et les possibilités d'échanges à courte distance; ce point devra être approfondi.

Les résultats fournis par les deux tests, biochimique et biologique, ne sont pas exactement superposables, surtout au mois de septembre, mais tous deux permettent de mettre en rapport une gamme de températures et une réaction du bourgeon. A notre connaissance, les données du test nucléotide présentées dans cette note constituent la première vérification biochimique, sur un végétal ligneux, des conceptions de VegIs (1964).

Les avantages de la technique biochimique mis en œuvre concernent essentiellement deux points : 1) il est possible de connaître quelques heures après la récolte du matériel végétal, donc beaucoup plus rapidement qu'avec les boutures de nœuds isolés, l'aptitude des bourgeons à la croissance ; 2) l'analyse porte sur le bourgeon seul, dégagé de toute corrélation avec l'axe dont le rôle modulateur de la croissance est connu (MENG-Horn et al., 1975).

Cependant la technique biochimique, telle qu'elle est actuellement employée, manque encore de précision. Chez les végétaux ligneux, elle ne permet que la détermination qualitative, et non quantitative, de l'aptitude d'un bourgeon à croître. Par ailleurs, un bourgeon est un ensemble complexe et les variations de teneur en NTP n'ont pas forcément toujours la même signification; il est permis de supposer que, selon les cas, la modification observée du métabolisme énergétique concerne les ébauches foliaires, ou les jeunes entre-nœuds, ou le méristème lui-même ou le bourgeon dans son entier. Il faut remarquer que la technique des boutures de nouds isolés présente le même type d’imprécision, car la détermination des débourrements telle qu'elle est faite ne permet pas de juger de l'aptitude à la croissance des divers territoires du bourgeon.

Un grand progrès sera fait dans la connaissance de la dormance des bourgeons de végétaux ligneux lorsque, comme cela a pu être déterminé dans le tubercule de crosne du Japon (TORT \& LAGARDE, 1981; TORT, 1977) on pourra préciser les optimums thermiques de croissance des diverses parties d'un bourgeon au cours de l'évolution de sa dormance. 


\subsection{Données nouvelles sur la dormance des bourgeons apicaux du frêne}

Les différences entre les résultats fournis par les deux tests, biochimique et biologiques, ont déjà été soulignées. Il convient maintenant de les discuter. Au mois de septembre le pool nucléotidique présente une régulation de type non dormant à $16^{\circ} \mathrm{C}$ et à $25^{\circ} \mathrm{C}$, alors que les bourgeons des boutures de nœuds isolés ne débourrent pas à $16^{\circ} \mathrm{C}$ et débourrent très mal à $25^{\circ} \mathrm{C}$. Tout se passe comme si le fragment d'axe porteur du bourgeon exerce un effet inhibiteur sur la croissance de celui-ci (LAvarenne et al., 1982). Cette inhibition qui entraîne le repos apparent au mois de septembre et, en conditions contrôlées, lors du repos apparent long de 15 mois de frênes placés à $25^{\circ} \mathrm{C}$ ou $12^{\circ} \mathrm{C}$ sous un jour long (LAvarenne-Barnola, publication en cours). Il apparaît que les corrélations entre l'axe et le bourgeon sont de la plus grande importance et leur caractérisation biochimique est actuellement à l'étude chez le châtaignier (PEZET, publication en cours).

Au mois de mars, la gamme des températures compatibles avec la croissance des bourgeons est ajustée aux caractéristiques thermiques du printemps des régions tempérées. Le débourrement des bourgeons des boutures de nœuds isolés est meilleur à $8^{\circ} \mathrm{C}$ et $16^{\circ} \mathrm{C}$ qu'à $25^{\circ} \mathrm{C}$ et les résultats du test biochimique indiquent une meilleure réactivité pour les deux premières températures, après carence partielle du matériel végétal. L'interrogation majeure concerne le décalage entre la date d'élimination de la dormance et celle du débourrement, dans les conditions naturelles, si la physiologie de l'eau dans l'apex (CotTignies) peut être invoquée, il est probable que d'autres modifications interviennent pendant cette période de post-dormance, chacune rendant le débourrement plus imminent. Des variations de $\mathrm{pH}$ intracellulaires, postérieures à la levée de dormance, antérieures au débourrement, ont été récemment mises en évidence (LAVARENNE, Barnola, publication en cours). Elles pourraient contribuer à restituer au bourgeon apical ses caractéristiques de "puits" (BARNOLA, LAVARFNnE, publication en cours).

De nombreuses études réalisées sur les végétaux ligneux ont eu pour objet la description, au cours de l'évolution de la dormance, de pools de métabolites en particulier de glucides (vigne : BouARd, 1966 ; peuplier : JOURDAN, 1980 ; BonICEL, 1985). Les variations enregistrées sont mises en parallèle avec les différentes phases de la dormance du végétal étudié. L'étude de la régularité du métabolisme nucléotidique présentée dans cette note ne relève pas de cette stratégie puisqu'elle fait appel, pour chaque période de la dormance, à l'analyse de la réaction du pool nulcotidique à un précurseur exogène. Cependant les mesures réalisées sur les bourgeons témoins peuvent, avec quelques précautions, être un reflet de l'état du pool des nucléotides au cours de l'évolution de la dormance. Vue sous cet angle, la période de dormance correspond à une contraction du pool des composés riches en énergie du bourgeon. Il serait alors intéressant de connaître la composition de l'ensemble désigné par NTP et connaître les teneurs en UTP, CTP, GTP, etc., en périodes de pré-dormance, de dormance et de post-dormance.

En conclusion, les résultats obtenus sur les bourgeons apicaux de frêne, montrent qu'il est possible chez un végétal ligneux de raisonner dormance et éventail de température selon VEGIS sur des caractéristiques biochimiques comme sur des critères phénologiques. La comparaison des techniques biochimiques et biologiques permet de faire la part des propriétés intrinsèques du bourgeon et du rôle des corrélations dans le contrôle du débourrement. 


\begin{abstract}
Summary
Apical bud dormancy of ash (Fraxinus excelsior. L) : an evaluation of the nucleotide triphosphate pool and the range of temperatures acting on bud opening during dormancy
\end{abstract}

The dormancy of buds of woody plants is usually studied using cuttings of isolated nodes. They are placed in controlled environment chambers where the temperatures are raised progressively from $5{ }^{\circ} \mathrm{C}$ to $30^{\circ} \mathrm{C}$. The more dormant the bud, the greater the range of temperatures within which growth is inhibited. It only opens at high temperatures. The breaking of dormancy is characterised by its opening at low temperatures. This idea, put forward by VEGIS (1964) has proved fruitful in the study of dormancy in trees and shrubs, but the biological technique used is questionable. The most important criticism, is that the length of the trial is sufficiently long that a new physiological state could arise in the cutting, different from that prevailing when it was sampled.

We suggest a new, biochemical technique based on the changes in cellular energy regulation, in relation to the state of dormancy. The ability of the plant material to increase its non-adenylated nucleotide triphosphates (NTP), following the introduction of an external precursor of ATP, adenosine, was measured. The results of this study are that the ideas of VeGis can be expressed biochemically for the first time. The optimum temperatures for the growth of the apical buds of ash at the end of a period of dormancy are defined more clearly than ever before.

Key words : Ash-tree, dormancy, nucleotide triphosphate pool.

\title{
Références bibliographiques
}

Arias O., Crabbé J., 1975. Les gradients morphogénétiques du rameau d'un an des végétaux ligneux, en repos apparent. Données complémentaires fournies par l'étude de Prunus avium L., Physiol. vég., 13 (1), 69-81.

Barnola P., 1970. Recherches sur le déterminisme de la basitonie chez le framboisier (Rubus idaeus L.). Ann. Sci. Nat. Bot., 11, 129-152.

BARNOLA P., 1976. Recherches sur la dormance et la morphogenèse de quelques espèces ligneuses buissonnantes. Thèse Doct. ès Sci. Nat. Univ. Clermont H, 153 p.

BONiCEL A., 1985. Les bases physiologiques de la culture du peuplier en taillis à courte rotation, Populus trichocarpa $\times$ Populus deltoïdes, clone Raspalje. Les variations saisonnières de l'aptitude au recépage et de la répartition des principaux sucres solubles et de l'amidon. Thèse Doct. ès Sci. Nat. Univ. Scientifique et Médicale, Grenoble.

BOUARD J., 1966. Recherches physiologiques sur la vigne et en particulier sur l'aoûtement des sarnents. Thèse Doct. ès Sci. Nat.. Univ., Bordeaux, 345 p.

Champagnat P., 1983. Quelques réflexions sur la dormance des bourgeons des végétaux ligneux. Physiol. vég., 21, 607-618.

Cotmignies A., 1983. Teneur en eau et dormance dans le bourgeon du frêne. Z. Pflanzenphysiol., 111, 133-139.

Crabbé J., 1968. Evolution annuelle de la capacité intrinsèque de débourrement des bourgeons successifs de la pousse de l'année chez le pommier et le poirier. Bull. Soc. Roy. Bot. Belgique, 101, 195-204.

CRocheт A., 1984. Essai de caractérisation de la dormance de jeunes plants de chênes pédonculés. D.E.A. Univ. Clermont II.

Gendraud M., 1977. Etude de quelques aspects du métabolisme des nucléotides des pousses de topinambour en relation avec leurs potentialités morphogénétiques. Physiol. vég., 15, 121-132.

Huglin P., 1958. Recherches sur les bourgeons de la vigne : initiation florale et développement végétatif. Thèse, Doct. ès Sci. Nat., Univ. Strasbourg, 174 p. 
Jourdan J., 1980. Variations saisonnières de la morphogenèse et de la croissance des systèmes aériens et souterrains chez le peuplier : leurs relations avec les glucides et les transferts minéraux. Thèse Doct. ès Sci. Nat. Univ. scientifique et médicale Grenoble.

Keppler D., Rudigifr J., Decker K., 1970. Enzymatic determination of uracil nucleotides in tissues. Analys. Biochem., 38, 105-114.

Lavarenne S., Barnola P., Champaginat P., 1980. Climats artificiels et dormance des bourgeons. I. Températures et dormance autommale chez le frêne (Fraxinus excelsior L.). Compte rendus Acad. Agric., 92-106.

Lavarenne S., Champciaux M., Barnola P., Gendraud M., 1982. Métabolisme des nucléotides et dormance des bourgeons chez le frêne. Physiol. vég., 20, 371-376.

Legave J.M., 1980. Contribution à l'étude du développement floral chez l'abricotier. Thèse Docteur Ingénieur. Univ. Sciences et techniques du Langucdoc, $167 \mathrm{p}$.

Mauget J.C., 1976. Sur la dormance des bourgeons végétatifs du noyer (Juglans regia L.). Compte rendus Acad. Sci. série D, 283, 499-502.

Meng Horn C., Champagnat P., Barnola P., Lavarenne S., 1975. L'axe caulinaire, facteur de préséances entre bourgeons sur le rameau de l'année du Rhamnus frangula L. Physiol. vég., 13, 335-348.

Monet R., Bastard Y., 1971. Dormance des bourgeons végétatifs de pêchers en période juvénile et d'arbres adultes (Prunus persica L. Batsch). Compte rendus Acal. Sci., D, 272, 2317-2319.

Nigond J., 1967. Recherches sur la dormance des bourgeons de la vigne. Thèse Doct. ès Sci. Nat., Paris, 153 p. Ann. Physiol. vég., 1, 2, 3, 4, 1967.

Povget R., 1963. Recherches physiologiques sur le repos de la vigne (Vitis vinifera L.) : la dormance des bourgeons et le mécanisme de sa disparition. Ann. Am. Pl., 13, numéro hors séric, 247 p.

Rageau R., 1978. Croissance et débourrement des bourgeons végétatifs de pêcher (Prunus persica L. Barsch) au cours d'un test classique de dormance. Compte rendus Acad. Sci., série $D, 287,1119-1122$.

Regnard J.L., 1984. Rôle de l'anoxie dans la levée de dormance des bourgeons de peuplier [Populus americana (DODE) GUINIER, C.U. 1214) : Analyse des processus physiologiques. Thèse Doct. 3" cycle, Univ. P.-et-M.-Curie, 151 p.

Si MOHAMid C., 1983. Germination, rythmes de croissance et morphogenèse de jeunes plants chez Castanea sativa Miller. Thèse Doct. 3" cycle, Univ. Clermont-Ferrand II, $201 \mathrm{p}$.

Sochet M.J., 1973. Contribution à l'étude de la dormance des bourgeons et ramifications du rameau d'un an de pommier. Thèse Doct. 3" cycle. Univ. Clermont II, 98 p.

Solignat G., 1977. Contribution à l'ćtude du débourrement végétatif chez le châtaignier (Castanea sp.). Pomol. Fr., XIX (10), 121-126.

Sovidi C., 1976. Recherches physiologiques sur la croissance et la morphogenèse des racines chez Populus $\times$ americana et Picca excelsa. Thèse Doct. $3^{\circ}$ cycle, Univ. Scicnces et Médicale Grenoble, $102 \mathrm{p}$.

Thomas F., TuÉvenor C., Gendraud M., 1985. Métabolisme des nucléosides tri-phosphates et dormance des cotylédons des embryons de pommier. Compte rendus Acad. Sci., Paris, 300, III, 9, 409-412.

TORT M., 1977. Aspects corrélatifs spatio cinétiques et cellulaires de la morphogenèse chez le crosne du Japon (Stachys sieboldi Miq.) cultivé à l'obscurité. Thèse Doct. ès Sci. Nat., Université Clermont II, 2 vol.

TORT M., LaGarde J., 1981. Etudes ultrastructurales chez le crosne du Japon cultivé en conditions contrôlées. Bull. Soc. bot. Fr., 128, Actual. bot., 2, 99-109.

Vegis A., 1964. Dormancy of higher plants. Ann. Rev. Plant. Physiol., 15, 185-224.

ZANETTE F., 1981. Recherches descriptives et expérimentales sur la morphogenèse des systèmes aériens et racinaires de quelques porte-greffes de pommier. Thèse Doct. Ingénieur. Univ. Clermont II, 159 p. 